\title{
Mareo residual, una condición clínica frecuente posterior a maniobra de reposición exitosa en vértigo posicional paroxístico benigno: Una revisión
}

\author{
Residual dizziness, a frequent clinical condition following successful \\ repositioning maneuver for benign paroxysmal positional vertigo: A review
}

Ignacio Novoa C', Carlos Pino U', Silvia Donoso T², Benjamín Romero B², Víctor Mercado M¹.

\begin{abstract}
RESUMEN
El vértigo posicional paroxístico benigno (VPPB) es reconocido como la principal causa de vértigo de origen periférico en adultos, ya que, si bien la etiología del VPPB aún no se ha demostrado plenamente y se clasifica como la mayoría de los casos, se puede identificar el desprendimiento de otolitos y su desplazamiento en uno de los tres canales semicirculares. Una anamnesis cuidadosa puede abordar el diagnóstico clínico del VPPB, pero la confirmación se obtendrá por medio de maniobras de diagnóstico específicas de acuerdo a cuál de los canales está involucrado. Este trastorno altera la capacidad de llevar a cabo actividades de la vida cotidiana que determinan un aumento significativo en el riesgo de caídas y las consiguientes lesiones. La mayoría de los pacientes se recuperan después del tratamiento, sin embargo, hasta dos tercios de éstos pueden percibir inestabilidad prolongada, aturdimiento y malestar definidos como mareo residual. Esta sintomatología residual después de la resolución del VPPB es variable entre los pacientes, siendo el tiempo de duración de los síntomas residuales de aproximadamente de 1 a 3 semanas, existiendo algunos pacientes que pueden recuperarse más tardíamente. En esta revisión analizaremos el mareo residual, describiendo su expresión clínica, diagnóstica, fisiopatológica y tratamientos actuales de esta entidad clínica.
\end{abstract}

Palabras clave: Vértigo posicional paroxístico benigno, maniobra de reposición, mareo residual, rehabilitación vestibular.

\begin{abstract}
Benign paroxysmal positional vertigo (BPPV) is recognized as the main cause of peripheral vertigo in adults, although the etiology of BPPV has not yet been fully demonstrated and is classified as idiopathicin most cases, detachment of otoliths and their
\end{abstract}

\footnotetext{
1 Instituto Neurobalance, Viña del Mar, Chile.

2 Clínica Red Salud, Vitacura, Santiago, Chile.

3 Universidad Santo Tomás, Valdivia, Chile.

Los autores declaran no tener conflictos de interés.

Recibido el 12 de septiembre de 2019. Aceptado el 12 de enero de 2020.
} 
displacement in one of the three semicircular canals can be identifie. A careful history can address the clinical diagnosis of BPPV, but confirmation will be obtained through specific diagnostic maneuvers according to which canal is involved. This disorder alters the ability to carry out activities of daily living that determine a significant increase in the risk of falls and consequent injuries. Most patients recover after treatment, however, up to two thirds of these patients may perceive prolonged instability, dizziness and discomfort defined as residual dizziness. This residual symptomatology following BPPV resolution is variable among patients, the duration of residual symptoms can last 1 to 3 weeks, with some patients may recover later on. In this review, we will analyze residual dizziness, describing its clinical presentation, diagnosis, pathophysiology and current treatments of this clinical entity.

Key words: Benign paroxysmal positional vertigo, maneuver, residual dizziness, vestibular rehabilitation.

\section{INTRODUCCIÓN}

El diagnóstico médico más frecuente en la clínica otoneurológica es el de vértigo posicional paroxístico benigno (VPPB) cuyo tratamiento de resolución gold standard es la maniobra de reposición de partículas (MRp) ${ }^{1}$. En un grupo importante de pacientes, las MRp solucionan los signos y los síntomas clínicos, no obstante, estudios publicados como el de Giommetti y cols describen que dos tercios de los pacientes sometidos a MRp exitosas presentan síntomas de mareo $^{2}$, definido como mareo residual (MR), ya que no presentan real vértigo ni presencia clínica de nistagmo, siendo descrito como una sensación subjetiva de mareo e inestabilidad. El reconocimiento y tratamiento temprano de pacientes con VPPB pueden disminuir la incidencia de MR ya que disminuirá la alteración de patrones normales de control postural e integración de los subsistemas sensoriales ${ }^{3,4}$. Diversas hipótesis se han planteado en el origen del $\mathrm{MR}^{5}$, además de cuáles serían las medidas terapéuticas.

\section{TEORÍAS DEL MAREO RESIDUAL}

\section{Duración del VPPB y factores de riesgo}

Vaduva y cols estudiaron 361 pacientes pos MRp y encontraron MR en 107 de ellos $(29,6 \%)$ sin diferencias significativas entre las tasas asociadas entre los tres canales semicirculares. El grupo que necesitó más de una MRp mostró una prevalencia significativamente mayor de MR que aquellos que requirieron una sola. El $47,2 \%$ de los pacientes con trastornos de ansiedad presentaron síntomas de MR. Frente a esto, se establecieron dos grupos de estudio, uno menor de 65 años y otro mayor de 65 años, siendo este último el que mostró un porcentaje significativamente mayor de MR que aquellos de menor edad 6 , lo que es compartido también por otros autores ${ }^{2,7-9}$. Ante esto, concluyen que posterior a un seguimiento de un mes de resuelto su VPPB, el MR puede asociarse con ciertos factores de riesgo, tales como (la edad, número de MRp necesarias, ansiedad y/o trastornos depresivos $)^{6}$.

Los síntomas referidos al MR pueden ser más frecuentes (30\%-61\%) durante los primeros días después del episodio agudo. Sin embargo, la mayoría de los pacientes se recuperan dentro de los 6 a 20 días después de la MRp. Un estudio realizado por von Brevern y cols en pacientes con canalolitiasis idiopática del canal posterior y horizontal refirieron que, la mitad de los pacientes que necesitaban más de una MRp presentaban MR, mientras que la prevalencia en aquellos que requieren sólo una, fue de 17,9\% ( $p=0,03)$, diferencia que podría explicarse por otoconias restantes entre MRp incompletas asociado a la recuperación tardía del VPPB ${ }^{10}$, llevando a un mayor tiempo necesario para la adaptación central después de MRp.

\section{Trastornos de ansiedad y trastornos depresivos}

Los pacientes que padecen trastornos de ansiedad y presentan un VPPB muestran MR más duraderos y más incapacitantes después de la resolución del vértigo agudo ${ }^{7}$. Mendel y cols informaron que, los 
pacientes con trastornos de ansiedad y depresión, presentaban estos síntomas persistentemente posteriores a la resolución de los trastornos vestibulares periféricos, generando una pérdida de confianza en sí mismos como en el riesgo de presentar caídas, lo que altera la vida diaria de los pacientes con esta condición. Sugiere además apoyo emocional continuo y un seguimiento a más largo plazo para los pacientes con VPPB asociado a ansiedad y/0 depresión ${ }^{11}$.

Los ansiolíticos pueden producir mareo, que probablemente contribuyan a la inestabilidad residual después de MRp efectivas en VPPB ya que, como se mostró en estudios previos ${ }^{7,11}$, los síntomas residuales fueron más prevalentes en el grupo de pacientes con ansiedad y depresión ${ }^{6}$. Dado que la ansiedad puede desempeñar un papel adicional en los mareos, es importante determinar la gravedad de ésta 0 el uso de escalas analógicas visuales, pudiendo éstas mejorar el valor de las conclusiones con respecto a la influencia de la ansiedad o la depresión en el VPPB ${ }^{6}$.

\section{Disfunción utricular}

Se plantea que, previo al VPPB idiopático, se podría presentar alguna patología como neuronitis vestibular 0 trauma encéfalo-craneano que generaría un mecanismo de daño en la matriz macular con posterior desprendimiento otoconial desde el utrículo a los canales semicirculares ${ }^{6}$. Estudios post mortem han resaltado signos de daño utricular unilateral en el lado afectado ${ }^{12-14}$. Dado que el VPPB se considera un trastorno macular, una disfunción utricular es una teoría posible como factor causal de MR. Un método simple para definir función utricular es el test vertical visual subjetivo (VVS) ${ }^{15,16}$. En la literatura, existen muchos resultados contradictorios sobre la VVS en pacientes con VPPB. Ejemplo de ello es el estudio de Bohmer y Rickenmann, donde examinaron a 19 pacientes con VPPB no tratados, sólo uno presentaba VV fuera del rango normal de $\pm 2^{\circ}$, inclinándose hacia el oído afectado ${ }^{16}$. En el caso de von Brevern y cols no encontraron diferencias en la percepción de VVS entre el grupo de pacientes y el grupo control ${ }^{10}$. Gall y cols describen desviaciones de la VVS en 14 de 16 pacientes con VPPB agudo en comparación con un grupo de control, pero no se informó si la VVS se desplazó hacia el oído afectado. Estos reportes dejan ver una posible disfunción otolítica en VPPB ${ }^{18}$, pero no se menciona correlación entre tales resultados y MR después de la MRp. Es así que Faralli y cols observaron sólo modificaciones subclínicas de la percepción de VVS en pacientes con inicio más reciente de VPPB. Estas alteraciones parecían ser rápidamente reversibles y en estos casos la disfunción otolítica podría explicar solo mareos breves después de la $M R p^{14}$, pero no MR más allá de una semana. Von Brevern y cols demostraron que una disfunción otolítica documentada en pacientes afectados por VPPB idiopático podría explicar el desequilibrio leve transitorio experimentado después de la resolución del vértigo agudo. La función otolítica también se evaluó mediante la respuesta del reflejo otolíticoocular, considerado más preciso en comparación con la VVS, donde se encontró disminución pocos días después del tratamiento en la amplitud del reflejo otolítico-ocular en pacientes con VPPB idiopático. Además, proporcionó evidencia que el VPPB idiopático se asoció con disfunción utricular de origen desconocido, posiblemente debido a un déficit de la matriz que adhiere la otoconia en la mácula, pudiendo ser ésta la causa del MR después de la MRp exitosa ${ }^{10}$.

Seo T y cols utilizaron potenciales miogénicos evocados vestibulares oculares (0-VEMP) para investigar la relación entre los MR y la función utricular después de MRp para el VPPB idiopático. Realizaron dichos potenciales en 40 pacientes con resultados exitosos de MRp, los que fueron examinados antes del tratamiento y una semana después de éste. Se analizaron varios factores generales y de los o-VEMP, arrojando que el MR se asoció significativamente con los resultados de la segunda prueba de 0 -VEMP ( $p=0,007)$, concluyendo que los MR posteriores a MRp exitosas pueden ser causados por una disfunción utricular persistente ${ }^{19}$.

\section{Reposición de otoconia y nuevo censo de la mácula}

En 2007, el editor de revista Auris Nasus Larynx revive una carta de Prokopakis y cols donde explica que en un estudio en 592 pacientes con MRp exitosa, 439 pacientes (74\%) relataban estar 
aturdidos y/0 mareados por las siguientes 48 a 72 horas. La teoría planteada en esta investigación se asocia con una nueva posición de las partículas removidas por la MRp dentro del vestíbulo de la mácula utricular, retomando una nueva posición hasta ahora desconocida, sugiriendo que debido a la nueva fijación de escombros en la membrana otolítica del utrículo ocurriría una adaptación a las señales recibidas ${ }^{20}$. Debido a esto, se produciría un cambio en la presión espacial de las otoconias, lo que conduciría a una alteración de la estimulación del epitelio sensorial del utrículo ${ }^{21}$.

\section{Disfunción simpático-neural}

Kim HA y cols estudian una asociación entre el MR después del tratamiento exitoso de pacientes con VPPB y la disfunción autonómica utilizando pruebas de función autónoma estandarizadas. En este estudio, aproximadamente el 35\% de los pacientes con VPPB que recibió un tratamiento exitoso, mostró al menos una anormalidad en la prueba de inclinación. Además, la incidencia de hipotensión ortostática durante la respuesta de inclinación y presión arterial anormal durante la maniobra de Valsalva, fue significativamente mayor en pacientes con MR que en aquellos sin esta condición. Estos hallazgos resaltan la importancia de la disfunción autonómica como una posible causa de MR después de un tratamiento exitoso en pacientes con VPPB y concluyen que el MR puede estar asociado, en parte, con disfunción autonómica simpático neural ${ }^{22}$. Se requiere más investigación de los mecanismos que subyacen a la asociación entre el MR y la disfunción autonómica, al igual que establecer si el MR es transversal a todas las causas de VPPB o solo en alguna en específico.

\section{ALGUNAS EVALUACIONES A CONSIDERAR}

Es importante en el MR evaluar los síntomas en el paciente, debido a la gran carga de ansiedad que provoca la inestabilidad inherente al VPPB. En pacientes adultos mayores, el componente emocional subjetivo evaluado en la escala Dizziness Handicap Inventory (DHI) se ve disminuido mayormente debido al síndrome poscaída o miedo a caer, quien juega un papel determinante en la génesis de la ansiedad relacionada con VPPB ${ }^{2,23}$. En un estudio en 258 pacientes con VPPB se evaluó el impacto del MR por medio de la escala de apoyo social, que se relaciona con el bienestar subjetivo y los efectos de la discapacidad autopercibida por medio de DHI. Dicho estudio concluyó que el apoyo social podría ayudar a reducir la incidencia de MR en pacientes con VPPB y volver a la vida diaria de manera más rápida y cómoda ${ }^{14,24}$. La escala visuoanalógica (VAS), que evalúa la presencia de MR, proporciona resultados consistentes al detectar diferentes aspectos relacionados con el vértigo, como el efecto posterior a las MRp o el rol del retraso de éstas, relacionando finalmente la puntuación del VAS con el bienestar percibido del paciente. La escala VAS es rápida, fácil de usar y supera barreras culturales e idiomáticas ${ }^{25}$, proporcionando una herramienta simple para la evaluación de mareos.

\section{TRATAMIENTOS ACTUALES PARA EL MAREO RESIDUAL}

\section{Rol de los medicamentos}

En relación al tratamiento médico en el control del MR, Swain y cols revelan que existen diferencias significativas en la duración del MR entre el grupo que se trató con ginkgo biloba (GB) (10-11 días) y el grupo sin GB (28-32 días). Los principales componentes de GB son los flavonoides y los terpenoides. La mejoría de la microcirculación y la propiedad antioxidante de GB puede ser la causa de controlar el MR después de la maniobra de MRp en pacientes con VPPB, concluyendo que el uso de GB puede minimizar significativamente el $\mathrm{MR}^{26}$. Acar y cols en un ensayo clínico aleatorio controlado, dividieron en cuatro grupos a los pacientes afectados por VPPB y que presentaban MR después MRp exitosas: un grupo no recibió medicación y los otros grupos recibieron betahistina, trimetazidina y GB, respectivamente. No encontraron diferencias significativas en las puntuaciones de $\mathrm{DHI}$ de premedicación de pacientes con MR entre los cuatro grupos ( $p>0,05$ ), lo que sugiere que la betahistina, la trimetazidina y el extracto de GB no alivian el MR²7. Otro estudio plantea que los supresores vestibulares 
redujeron significativamente los síntomas residuales en comparación con el grupo control posterior a la MRp. Sin embargo, no se encontró una reducción significativa en la puntuación de DHI en comparación con el grupo de control. A partir de esto, el autor sugiere que los síntomas residuales no se pueden evaluar solo con la puntuación de $\mathrm{DH}{ }^{28}$.

Wan realizó un estudio en 50 pacientes que presentaban MR asociando bajas dosis de betahistina (6 mg cada 8 horas) a una terapia cognitiva conductual (TCC), demostró que dosis bajas de betahistina más TCC deben recomendarse por sobre dosis altas de betahistina en el tratamiento del $M^{29}$. Jung y cols sugieren en su estudio, el uso de ansiolíticos en el MR con una dosis baja de etizolam, basado en la similitud de los síntomas del MR con los de la ansiedad (frecuente en este grupo de pacientes). Este estudio mostró una resolución en la puntuación total de DHI y, en particular, en las subescalas funcionales y emocionales, permitiendo un regreso más rápido y más cómodo a la vida diaria ${ }^{30}$. Hesse $\mathrm{G}$, plantea respecto del estudio de Jung y $\operatorname{cols}^{30}$ que se requieren más estudios de protocolos controlados con placebo para afirmar que el uso de etizolam en pacientes con MR pueda ser una alternativa terapéutica válida ${ }^{31}$.

\section{Rol de la rehabilitación vestibular}

Variados estudios han tenido el objetivo de comprobar que la RV pos MRp es efectiva en dar una adecuada solución al MR, entre ellos el realizado por Ribeiro y cols el cual mostró los efectos de la RV en el equilibrio postural en personas mayores con VPPB, incluyendo diversos ejercicios. Se encontró que el grupo que realizó la RV mejoró el equilibrio dinámico en comparación con el grupo de control que realizó solo MRp ${ }^{32}$. Similares resultados fueron encontrados en pacientes posterior a RV con VPPB asociado a un traumatismo encéfalo craneano ${ }^{33}$. Resende y cols crearon un protocolo de RV realizando dos sesiones semanales hasta completar 5 semanas de tratamiento en pacientes geriátricos con VPPB, concluyendo que es una excelente estrategia terapéutica ya que no solo se obtiene resolución de los síntomas, sino que además promovió una mejoría en sus aspectos sociales y psicológicos ${ }^{34}$.
Ding y cols examinaron el efecto terapéutico de la RV sobre el MR después de MRp exitosas en pacientes de edad avanzada. Trataron 86 pacientes con MR usando método de tabla aleatoria, 43 casos en un grupo de observación y 43 en un grupo de control. Ambos fueron tratados con dioxetina mesilato, y el grupo de observación recibió además RV. Se comparó el efecto curativo utilizando la escala de equilibrio de Berg, la puntuación del índice de síntomas vestibulares (VSI) y la puntuación de discapacidad de mareos (DHI). Los resultados concluyen que la RV es efectiva para el MR en pacientes de edad avanzada con VPPB35. Xu y cols estudiaron 93 pacientes que fueron diagnosticados con VPPB quienes luego de realizar MRp todavía presentaban síntomas residuales. Se constituyeron grupos de manera aleatoria: el grupo de control (A); el grupo de medicamentos (cápsulas de gel de hojas de ginkgo y tabletas de Qiangli Dingxuan B, una mezcla de plantas); y el grupo de RV (C). El seguimiento de 30 días evidenció una disminución estadísticamente significativa de las puntuaciones de $\mathrm{DHI}$ del grupo $A$ con respecto al grupo $B(p<0,05)$ y $C(p<0,05)$. El tiempo de duración del MR del grupo A muestra una diferencia significativa con el grupo $B(p<0,05)$ y C $(p<0,05)$. No hubo diferencias estadísticamente significativas al comparar los puntajes de DHI en el seguimiento de 30 días, y la duración del MR de grupos $B$ y $C(p>0,05)$. Los medicamentos para mejorar la microcirculación y la RV podrían reducir la duración de los síntomas asociados al MR².

Sun y cols estudiaron el efecto curativo y la calidad de vida del entrenamiento de RV sobre el MR después de MRp exitosas en pacientes con VPPB. Los pacientes con MR se distribuyeron de manera aleatoria en tres grupos: grupo de control A con 32 pacientes; grupo de intervención $B$, el cual fue guiado a un entrenamiento de autorrehabilitación que incluyó a 33 pacientes; y el grupo $\mathrm{C}$ que tuvo entrenamiento guiado con ejercicios de Brandt-Daroff que incluyó a 33 pacientes. El DHI y MR se utilizaron como método de evaluación, después de una semana de entrenamiento de RV las puntuaciones de $\mathrm{DHI}$ en el grupo $\mathrm{B}$ y $\mathrm{C}$ disminuyeron, y no hubo diferencias significativas entre ellos $(p>0,05)$. Después de cuatro semanas de entrenamiento de RV las puntuaciones de $\mathrm{DH}$ en el grupo $\mathrm{B}$ y el grupo $\mathrm{C}$ fueron más bajas que las del grupo $A$, la disminución del grupo $B$ fue significativamente mayor que la del 
grupo $C(p<0,01)$. Concluyen que el entrenamiento adecuado de RV en MR después de MRp exitosas en pacientes con VPPB puede ayudar a promover la compensación vestibular central, reducir los síntomas residuales, mejorar la calidad de vida de los pacientes y puede usarse como tratamiento adyuvante en pacientes con síntomas de $\mathrm{MR}^{37}$.

En un ensayo clínico aleatorizado realizado por Chang y cols encuentran que las personas con VPPB que fueron tratadas con MRp y ejercicios adicionales de RV (equilibrio/habituación) habían mejorado significativamente el control postural por sobre quienes recibieron MRp (Epley) aislada por su VPPB. Además, este estudio documentó que se logró un mayor equilibrio en los pacientes sólo cuando se administró RV adicional basada en el movimiento/habituación ${ }^{38}$.

\section{BIBLIOGRAFÍA}

1. Bhattacharyya N, Gubbels SP, Schwartz SR, et al. Clinical practice guideline: benign paroxysmal positional vertigo (Update). Otolaryngol Head Neck Surg 2017; 156: S1-S47.

2. Giommetti G, Lapenna R, Panichi R et al. Residual Dizziness after Successful Repositioning Maneuver for Idiopathic Benign Paroxysmal Positional Vertigo: A Review. Audiol Res 2017; 7: 31-7.

3. Seok JI, Lee HM, Yoo JH, Lee DK. Residual dizziness after successful repositioning treatment in patients with benign paroxysmal positional vertigo. J Clin Neurol2008; 4: 107-10.

4. Stambolieva K, Angov G. Postural stability in patients with different durations of benign paroxysmal positional vertigo. Eur Arch Otorhinolaryngol 2006; 263: 118-22.

5. Teggl R and Nutı D. Residual Dizziness after Physical Treatment for Benign Paroxysmal Positional Vertigo. A Review of Recent Literature. EC Neurology 2017; 7: 158-64.

6. Vaduva C, Esteban-Sánchez J, Sanz-Fernández R, Martín-Sanz E. Prevalence and management of post-BPPV residual symptoms. Eur Arch Otorhinolaryngol 2018; 275: 1429-37.

7. Martellucci S, Pagliuca G, de Vincentils M et al. Features of residual dizziness after canalith

\section{CONCLUSIÓN}

El MR es una condición clínica frecuente en pacientes con VPPB idiopático, aun posterior a una MRp resolutiva/efectiva y no parece estar relacionado con alteraciones otolíticas, las que son tempranas, moderadas, fugaces y rápidamente reversibles. Esta condición clínica se debería a una modificación de los mecanismos de control postural y balance, lo que conduce a que el individuo deba readaptarse posterior a la MRp. El tratamiento temprano de VPPB sería una acción preventiva en el MR, especialmente en pacientes con trastornos de ansiedad y adultos mayores. La RV pos MRp es un aporte en la recuperación del balance, mejorando la integración sensorial y disminuyendo el riesgo de caídas.

repositioning procedures for benign paroxysmal positional vertigo. Otolaryngol Head Neck Surg 2016; 154: 693-701.

8. Batuecas-Caletrio A, Trinidad-Ruiz G, Zschaeck C, ET AL. Benign paroxysmal positional vertigo in the elderly. Gerontology 2013; 59: 408-12.

9. Teggi R, Giordano L, Bondi S, Fabiano B, Bussi M. Residual dizziness after successful repositioning maneuvers for idiopathic benign paroxysmal positional vertigo in the elderly. Eur Arch Otolaryngol 2011; 268: 507-11.

10. Von Brevern M, Schmidt T, Schönfeld U, Lempert T, ClaRke AH. Utricular dysfunction in patients with benign paroxysmal positional vertigo. Otol Neurotol 2006; 27: 92-6.

11. Mendel B, Bergenius J, Langius A. Dizziness symptom severity and impact on daily living as perceived by patients suffering from peripheral vestibular disorder. Clin Otolaryngol Allied Sci 1999; 24: 286-93.

12. Cawthorne TE, Hallpike CS. A study of the clinical features and pathologic changes within the temporal bones, brainstem and cerebellum of an early case of positional nystagmus of the so called benign paroxysmal type. Acta Otolaryngol 1957; 48: 89-105.

13. LindSAY JR, Hemenway WG. Postural vertigo due to unilateral sudden partial loss of vestibular function. Ann Otol Rhinol Laryngol 1956; 65: 692-706. 
14. Faralli M, Lapenna R, Giommetti G, Pellegrino C, Rıcc G. Residual dizziness after the first BPPV episode: role of otolithic function and of a delayed diagnosis. Eur Arch Oto Rhino Laryngol 2016; 273: 3157-65.

15. Oliva C,Ochoa N, Kuroiwa M,Barraza C, Núñez M, Breinbauer H. Nuevo método para evaluar el Test Subjetivo Vertical. Rev Otorrinolaringol Cir Cabeza Cuello 2017; 77: 124-34.

16. Halmagy GM, Curthors IS. Clinical testing of otolith function. Ann NY Acad Sci 1999; 871: 195-204.

17. Bohmer A, Rickenmann J. The Subjective visual vertical as a clinical parameter of vestibular function in peripheral vestibular diseases. $J$ Vestib Res 1995; 5: 35-45.

18. Gall RM, Ireland DJ, Robertson DD. Subjective visual vertical in patients with benign paroxysmal positional vertigo. J Otolaryngo/ 1999; 28: 162-5.

19. Seo T, Shiraishi K, Kobayashi T, Mutsukazu K et al. Residual dizziness after successful treatment of idiopathic benign paroxysmal positional vertigo originates from persistent utricular dysfunction. Acta Otolaryngol 2017; 137: 1149-52.

20. Prokopakis EP, Lachanas Va, Christodoulou PN, Velegrakis GA. Dizziness after canalith repositioning procedure for benign paroxysmal positional vertigo. Auris Nasus Larynx 2007; 35: 435.

21. Prokopakis eP, Chimona T, Tsagournisakis M, Christodoulou P, Hirsch BE, Lachanas VA, et al. Benign paroxysmal positional vertigo: 10-year experience in treating 592 patients with canalith repositioning procedure. Laryngoscope 2005; 115: 1667-71.

22. KIm HA, LeE H. Autonomic dysfunction as a possible cause of residual dizziness after successful treatment in benign paroxysmal positional vertigo. Clin Neurophysiol2014; 125: 608-14.

23. Lee NH, Kwon HJ, Ban JH. Analysis of Residual Symptoms after Treatment in Benign Paroxysmal Positional Vertigo Using Questionnaire. Otolaryngol Head Neck Surg 2009; 141: 232-6.

24. Duan Z, Fu C, Tang T, Liu B, Liu J, Chen B, Zhang $X$. Social support reduces residual dizziness after canalith repositioning procedure in benign paroxysmal positional vertigo. Biomed Res 2018; 29: 1988-93.
25. Toupet M, Ferrary E, Grayell AB. Visual analog scale to assess vertigo and dizziness after repositioning maneuvers for benign paroxysmal positional vertigo. J Vest Res 2011; 2: 235-41.

26. Swain SK, Behera IC, Sahu MC. Role of Ginkgo biloba for controlling residual dizziness after successful treatment of benign paroxysmal positional vertigo: Our experiences at a tertiary care teaching hospital of Eastern India. Int $J$ Health Allied Sci 2018; 7: 196-200.

27. Acar B, Karasen RM, Buran Y. Efficacy of medical therapy in the prevention of residual dizziness after successful repositioning maneuvers for benign paroxysmal positional vertigo (BPPV). B-ENT 2015; 11: 117-21.

28. KIM MB, LeE HS, BAN JH. Vestibular suppressants after canalith repositioning in benign paroxysmal positional vertigo. Laryngoscope 2014; 124 : 2400-3.

29. Wan TJ, Yu YC, Zhao XG, Tang P, Gong YS. Efficacy of betahistine plus cognitive behavioral therapy on residual dizziness after successful canalith repositioning procedure for benign paroxysmal positional vertigo. Neuropsychiatr Dis Treat 2018; 14: 2965-71.

30. Jung HJ, Koo JW, KIm CS, KIm JS, Song JJ. Anxiolytics reduce residual dizziness after successful canalith repositioning maneuvers in benign paroxysmal positional vertigo. Acta Oto Laryngol 2012; 132: 277-84.

31. HeSSE G. Anxiolytics do not reduce dizziness, they reduce fear and have harmful side effects! Acta Otolaryngol 2012; 132: 903.

32. Ribeiro KF, Oliveira BS, Freitas RV, Ferreira LM, Deshpande N, Guerra Ro. Effectiveness of Otolith Repositioning Maneuvers and Vestibular Rehabilitation exercises in elderly people with benign paroxysmal positional vertigo: a systematic review. Braz J Otorhinolaryngo/2018; 84: 109-18.

33. Alsalaheen BA, Whitney SL, Mucha A, Morris LO, Furman JM, Sparto PJ. Exercise prescription patterns in patients treated with vestibular rehabilitation after concussion. Physiother Res Int 2013; 18: 100-8.

34. Resende C, Taguchi C, de Almeida J, Fujita R. Vestibular rehabilitation in elderly patients with benign paroxysmal positional vertigo. Braz $J$ Otorhinolaryngol 2003; 69: 34-8. 
35. DING J, LIU Y. Effect of vestibular rehabilitation on residual dizziness in elderly patients with benign paroxysmal positional vertigo. J Otolaryngol Ophthalmol Shandong Univ 2018; 32: 64-8.

36. Xu Y, Fu M, Zhang N. Lin Chung Er Bi Yan Hou Tou Jing Wai Ke Za Zhi 2016; 30: 1146-49. doi:10.13201/j.issn.1001-1781.2016.14.015.
37. Sun LB, Zheng ZY, Wang BQ, et al. Lin Chung Er Bi Yan Hou Tou Jing Wai Ke Za Zhi 2017; 31: 897-900. doi:10.13201/j.issn.10011781.2017.12.001.

38. Chang WC, Yang YR, Hsu LC, et al. Balance improvement in patients with benign paroxysmal positional vertigo. Clin Rehabil2008; 22: 338-47.

Correspondencia: Ignacio Novoa C. Instituto Neurobalance Avenida Libertad 798, oficina 901. Viña del Mar, Chile

E mail: ignacio.novoa.c@gmail.com 